\title{
A CLASS OF DIFFERENTIAL FIELDS WITH MINIMAL DIFFERENTIAL CLOSURES
}

\author{
MICHAEL F. SINGER
}

\begin{abstract}
We give examples of differential fields which are not differentially closed but which become differentially closed when one adjoins $\sqrt{-1}$; differential fields whose differential closures are therefore minimal.
\end{abstract}

Kolchin [1], Rosenlicht [2], and Shelah [6] have shown that the differential closure of the rational numbers is not minimal. It is not generally known (cf. [8, p. 350]), though, that there are nontrivial examples of differential fields with minimal differential closures. In this note we show that there is a class of differential fields, the closed ordered differential fields, which are not differentially closed but which become differentially closed when one adjoins $\sqrt{-1}$. This will give us the desired examples. I would like to thank $\mathrm{C}$. Wood for conversations which stimulated the work in this note.

We will assume that the reader is familiar with the model theory of differential fields ([3], [8]). We only recall that a differentially closed field of characteristic 0 is a differential field of characteristic 0 which is algebraically closed and which has, for every pair of differential polynomials $f(y), g(y)$ with ord $f>$ ord $g$, a solution of $f(y)=0, g(y) \neq 0$. An ordered (real) differential field is a differential field which is also an ordered (real) field. In [7], we showed that the theory of ordered differential fields has a model completion called the theory of closed ordered differential fields. A closed ordered differential field $K$ is a differential field which is also a real closed ordered field and which satisfies the following property: let $f(y)$, $g_{1}(y), \ldots, g_{m}(y)$ be differential polynomials such that $n=$ ord $f \geqslant$ ord $g_{i}$ for $i=1, \ldots, m$. If there exist elements $c_{0}, \ldots, c_{n}$ in $K$ such that $f\left(c_{0}, \ldots, c_{n}\right)$ $=0, \quad\left(\partial f / \partial y^{(n)}\right)\left(c_{0}, \ldots, c_{n}\right) \neq 0$ and $g_{i}\left(c_{0}, \ldots, c_{n}\right)>0$ (here we are considering these polynomials as algebraic polynomials and substituting $c_{i}$ for $\left.y^{(i)}\right)$ then there is a $z$ in $K$ such that $f(z)=0, g_{i}(z)>0$ for $i=1, \ldots, m$ (here the polynomials are considered differential polynomials). In the theorem below we will use two embedding theorems. The first one, whose proof appears in [7], states that if $K=Q\left\langle u_{1}, \ldots, u_{n}\right\rangle$ is a real differential extension of the rationals $Q$, then $K$ is isomorphic to $Q\left\langle\bar{u}_{1}, \ldots, \bar{u}_{n}\right\rangle$ where each $\bar{u}_{i}$ is a real analytic function of $x$, defined in some neighborhood of 0

Received by the editors October 5, 1977.

AMS (MOS) subject classifications (1970). Primary 02H15, $12 \mathrm{H} 05$.

(c) American Mathematical Society 1978 
and where the derivation on $Q\left\langle u_{1}, \ldots, u_{n}\right\rangle$ is $d / d x$. The second theorem, due to Seidenberg, [4] and [5], states that if $K \subset K_{1}$ are finitely generated differential extensions of the rational numbers and $\tau: K \rightarrow K^{*}$ is an isomorphism of $K$ onto a field of meromorphic functions on some complex domain $\mathcal{G}$, then $\tau$ can be extended to an isomorphism $\tau_{1}$ of $K_{1}$ onto $K_{1}^{*}$, a field of meromorphic functions on a domain $\mathcal{G}_{1} \subset \mathcal{G}$. Furthermore, given $K$ and $K_{1}$, there are at most a countable number of points $\left\{\alpha_{i}\right\}$ in $\mathcal{S}$ such that given any $\alpha$ in $\mathcal{G}$, not equal to some $\alpha_{i}$, we can choose $\mathcal{S}_{1}$ to be an open disk around $\alpha$.

We can now show

THEOREM. If $K$ is a closed ordered differential field, then $K(i)$ is a differentially closed field of characteristic 0 , where $i=\sqrt{-1}$.

Proof. Since $K$ is real closed, $K(i)$ is an algebraically closed field of characteristic 0 . Therefore, to verify the conclusion, we need only show that if $f(y)$ and $g(y)$ are differential polynomials with coefficients in $K(i)$ and ord $f>$ ord $g$, then $f=0, g \neq 0$ has a solution in $K(i)$. Substitute $y_{1}+i y_{2}$ for $y$ (where $y_{1}$ and $y_{2}$ are new differential indeterminants) and write $f\left(y_{1}+\right.$ $\left.i y_{2}\right)=f_{1}\left(y_{1}, y_{2}\right)+i f_{2}\left(y_{1}, y_{2}\right)$ and $g\left(y_{1}+i y_{2}\right)=g_{1}\left(y_{1}, y_{2}\right)+i g_{2}\left(y_{1}, y_{2}\right)$ where $f_{1}, f_{2}, g_{1}, g_{2}$ have coefficients in $K$. Let $\bar{f}\left(y_{1}, y_{2}\right)=f_{1}^{2}+f_{2}^{2}$ and $\bar{g}\left(y_{1}, y_{2}\right)=g_{1}^{2}$ $+g_{2}^{2}$. If $F$ is any real differential extension of $k$ (the smallest subfield of $K$ containing the coefficients of $\left.f_{1}, f_{2}, g_{1}, g_{2}\right)$ and $u_{1}, u_{2}$ are members of $F$, we then have that $\bar{f}\left(u_{1}, u_{2}\right)=0, \bar{g}\left(u_{1}, u_{2}\right) \neq 0$ if and only if $f(u)=0, g(u) \neq 0$ in $F(i)$ where $u=u_{1}+i u_{2}$. We now claim that ODF $\cup \mathcal{D}(K) \cup\left\{\exists y_{1}, y_{2}\right.$ $\left.\left(\bar{f}\left(y_{1}, y_{2}\right)=0, \bar{g}\left(y_{1}, y_{2}\right) \neq 0\right)\right\}$ is consistent (where ODF is the theory of ordered differential fields and $\mathscr{D}(K)$ is the diagram of $K$ ). Assuming this for a moment, we can finish the proof of the proposition. Let $F$ be a model of these formulas and embed $F$ in a closed ordered differential field $F^{\prime} . F$ contains a copy of $K$, and since the theory of closed ordered differential fields is model complete, $K$ must be an elementary submodel of $F^{\prime}$. Therefore there must exist a $u_{1}$ and $u_{2}$ in $K$ such that $\bar{f}\left(u_{1}, u_{2}\right)=0$ and $\bar{g}\left(u_{1}, u_{2}\right) \neq 0$. This implies $f(y)=0, g(y) \neq 0$ has a solution in $K(i)$.

To prove the claim we will use the compactness theorem. Let $S$ be a finite subset of $\mathscr{D}(K)$. We will show that ODF $\cup S \cup\left\{\exists y_{1}, y_{2} \bar{f}\left(y_{1}, y_{2}\right)=0\right.$, $\left.\bar{g}\left(y_{1}, y_{2}\right) \neq 0\right\}$ has a model. We can assume $S$ contains terms representing the coefficients of $f_{1}, f_{2}, g_{1}$ and $g_{2}$. We can also assume that any use of the symbol " $>$ " in $S$ occurs as " $a>0$ " where $a$ is some term. Take atomic formulas in $S$ of the form " $a>0$ " and replace them by formulas " $a=b^{2}$ " and " $b \neq 0$ " for some term $b$. We can do this since $K$ is real closed. Let $S^{*}$ be the resulting set of formulas after we have done all of this. $S^{*}$ has no occurrence of " $>$ ". We will show that RDF $\cup S^{*} \cup\left\{\exists y_{1}, y_{2}(\bar{f}=0, \bar{g} \neq 0)\right\}$ has a model $E$, (where RDF is the theory of real differential fields). This $E$ would then be a real field and therefore orderable. Any ordering of $E$ will satisfy the formulas " $a>0$ " in $S$ since $a$ will have a nonzero square root in $E$. Thus, any ordering on $E$ 
will turn $E$ into a model of ODF $\cup S \cup\left\{\exists y_{1}, y_{2}(\bar{f}=0, \bar{g} \neq 0)\right\}$. The reason for switching to real fields instead of dealing directly with ordered fields is that we can now use the first representation theorem. To see that RDF $\cup S^{*}$ $\cup\left\{\exists y_{1}, y_{2}(\bar{f}=0, \bar{g} \neq 0)\right\}$ has a model, let $k$ be the smallest differential subfield of $K$ containing elements representing all the terms mentioned in $S^{*}$ and the coefficients of $f_{1}, f_{2}, g_{1}$ and $g_{2}$. Since $K$ can be ordered, this is a real differential field. Since it is finitely generated over $Q$, it is isomorphic (by the first embedding theorem mentioned above) to a field $\tilde{k}$ of real functions, meromorphic in some real neighborhood of 0 . We can formally extend $k$ to a differential field (not necessarily real) $k\langle y\rangle$ containing a solution of $f=0$, $g \neq 0$. $\tilde{k}$ can also be considered a field of meromorphic functions in some complex neighborhood $\mathcal{G}$ of zero. Using the second embedding theorem, we can extend the isomorphism of $k$ onto $\tilde{k}$ to an isomorphism of $k\langle y\rangle$ onto $\tilde{k}\langle\tilde{y}\rangle$, where $\tilde{y}$ is analytic in some smaller open set $\mathcal{G}^{\prime} \subset \mathcal{G}$ and where $\mathcal{G}^{\prime}$ can be chosen to contain a point $\alpha$ on the real axis. Any function $h(z)$, meromorphic at $\alpha$, can be written as $h(z)=h_{1}(z)+i h_{2}(z)$ where $h_{1}$ and $h_{2}$ are real meromorphic functions. Note that if $h \in \tilde{k}$ then $h=h_{1}+i h_{2}$ where $h_{2} \equiv 0$. Let $E$ be the differential field generated by the $h_{1}, h_{2}$ corresponding to all $h$ in $\tilde{k}\langle\tilde{y}\rangle . E$ is a real field since it can be considered a subfield of $Q((x-\alpha))$ and by the above, $E$ contains a copy of $k$. If we write $\tilde{y}=y_{1}+$ $i y_{2}$, then $y_{1}$ and $y_{2}$ are in $E$ and $\bar{f}\left(y_{1}, y_{2}\right)=0, \bar{g}\left(y_{1}, y_{2}\right) \neq 0$, so $E$ is a model of $\mathrm{RDF} \cup S^{*} \cup\left\{\exists y_{1}, y_{2}\left(\bar{f}\left(y_{1}, y_{2}\right)=0, \bar{g}\left(y_{1}, y_{2}\right) \neq 0\right)\right\}$. This concludes the proof.

It is now easy to see that any closed ordered differential field $K$ has a minimal differential closure, since $K(i)$ is differentially closed and there are no fields properly between $K$ and $K(i)$. This fact suggests two questions. First, precisely which differential fields have minimal differential closures? Secondly, what characterizes the class of fields of finite codimension in their differential closures, i.e. which differential fields $K$ have the property that $[\tilde{K}: K]$ is finite (and therefore equal to 2 ), where $\tilde{K}$ is the differential closure of $K$ ? Both classes contain the differentially closed fields and the closed ordered differential fields, but I do not know if either class contains more fields or if the classes are different. On the other hand, the second class of differential fields mentioned above does coincide with the class of differential fields $K$ which have the property that the differential closure of $K$ is finitely generated (in the differential sense) over $K$. Clearly, the latter class contains the former. To see that the former class contains the latter, let $K$ be a differential field, $\tilde{K}$ its differential closure and say $\tilde{K}=K\left\langle y_{1}, \ldots, y_{n}\right\rangle$. I will show that the $y_{i}$ 's must be algebraic over $K$. If the $y_{i}$ 's were not algebraic over $K$, we could then find a field $L$ (not necessarily a differential field) so that $L$ would be a pure transcendental extension of $K$ and $\tilde{K}$ would be a finite algebraic extension of $L$. If $u$ were an element of $L$, not in $K$, then $L$ could not possibly contain roots of $X^{n}-u$ for all $n$. $\tilde{K}$ would not be algebraically closed and therefore, wơuld not be differentially closed, a contradiction. 


\section{REFERENCES}

1. E. Kolchin, Constrained extensions of differential fields, Advances in Math. 12 (1974), 141-170.

2. M. Rosenlicht, The nonminimality of the differential closure, Pacific J. Math. 52 (1974), 529-537.

3. G. Sacks, Saturated model theory, Benjamin, Reading, Mass., 1972.

4. A. Seidenberg, Abstract differential algebra and the analytic case, Proc. Amer. Math. Soc. 9 (1958), 159-164.

5. Abstract differential algebra and the analytic case. II, Proc. Amer. Math. Soc. 23 (1969), 689-691.

6. S. Shelah, Differentially closed fields, Israel J. Math. 16 (1973), 314-328.

7. M. Singer, The model theory of ordered differential fields, J. Symbolic Logic (to appear).

8. C. Wood, The model theory of differential fields revisited, Israel J. Math. 25 (1976), 331-352.

Department of mathematics, North Carolina State University, Raleigh, North Carolina 27607 